\title{
Dyrk1A Influences Neuronal Morphogenesis Through Regulation of Cytoskeletal Dynamics in Mammalian Cortical Neurons
}

\author{
M. Martinez de Lagran , R. Benavides-Piccione, I. Ballesteros-Yañez , M. Calvo , M. Morales ,C. Fillat , J. DeFelipe ,
} G. J. A. Ramakers and M. Dierssen

\begin{abstract}
Down syndrome (DS) is the most frequent genetic cause of mental retardation. Cognitive dysfunction in these patients is correlated with reduced dendritic branching and complexity, along with fewer spines of abnormal shape that characterize the cortical neuronal profile of DS. DS phenotypes are caused by the disruptive effect of specific trisomic genes. Here, we report that overexpression of dual-specificity tyrosine phosphorylationregulated kinase 1A, DYRK1A, is sufficient to produce the dendritic alterations observed in DS patients. Engineered changes in Dyrk1A gene dosage in vivo strongly alter the postnatal dendritic arborization processes with a similar progression than in humans. In cultured mammalian cortical neurons, we determined a reduction of neurite outgrowth and synaptogenesis. The mechanism underlying neurite dysgenesia involves changes in the dynamic reorganization of the cytoskeleton.
\end{abstract}

Keywords: actin cytoskeleton, Down syndrome, Dyrk1A, mental retardation, neuritogenesis, synaptogenesis

\section{Introduction}

Dendritic abnormalities are a common feature of the microstructural pathology of mental retardation. Down syndrome (DS) is the most common genetic form of mental retardation, and DS patients show reduced dendritic branching and complexity along with fewer spines of abnormal shape. However, DS fetus and newborn present normal or even increased dendritic branching (see Becker et al. 1986; Dierssen and Martinez de Lagran 2006 for review).

$D Y R K 1 A$ is a plausible candidate gene to explain some DS phenotypes (reviewed in Dierssen and Ramakers 2006; Dierssen et al. 2009). It localizes in the DS critical region of human chromosome 21 , encodes for a dual kinase phosphorylating both threonine/serine and tyrosine residues, and has been implicated in learning and memory (Altafaj et al. 2001; Ahn et al. 2006). Interestingly, Dyrk $1 \mathrm{~A}+/-$ mice show microcephaly and dendritic defects (Fotaki et al. 2002; BenavidesPiccione et al. 2005) and DYRK1A knockdown in cortical cultures compromises neuritogenesis (Scales et al. 2009). Moreover, primary cultures of transgenic mice ( $\mathrm{Tg} 152 \mathrm{~F} 7)$ that contain a $570 \mathrm{~kb}$ human YAC, including DYRK1A show dendritic growth impairment (Lepagnol-Bestel et al. 2009).

Here, we demonstrate that single overexpression of Dyrk1A in vivo to the levels observed in DS (1.5-fold in brain) is sufficient to recapitulate the dendritic features of DS patients by affecting neurite outgrowth, synaptogenesis, and actin dynamics within dendritic spines.

\section{Materials and Methods}

\section{Animals}

The production and breeding procedures of transgenic mice for Dyrk1A (TgDyrk1A) are described in Altafaj et al. (2001). Animals were housed under standard conditions and protocols, and experiments were performed according with local and EU Ethical Committee guidelines.

\section{Intracellular Injections of Cortical Pyramidal Cells}

Four to six mice per genotype of different postnatal stages (postnatal days P7 and P10) and adults ( 2 months) were used to study pyramidal cell morphology. Intracellular injections of Lucifer yellow (LY) were performed in layer $\mathrm{III}$ pyramidal neurons of the secondary motor cortex (M2; Paxinos and Franklin 1997). To sample a homogeneous collection of dendritic arbors, the whole basal dendritic field of pyramidal neurons, that forms about $90 \%$ of the dendritic length of any cortical pyramidal neuron (Larkman 1991) and, thus, represents the major target of synaptic inputs to pyramidal neurons, was studied in horizontal sections. The M2 region was selected to compare the results of dendritic morphology with those of other DS animal models such as Ts65Dn and Dyrk1A+/- (Dierssen et al. 2003; BenavidesPiccione et al. 2005). Animals were perfused with $4 \%$ paraformaldehyde (PFA), and their brains vibratome sliced parallel to the cortical surface in $150-\mu \mathrm{m}$ sections. Cell injection methodology was previously described in detail (Elston and Rosa 1997; Elston et al. 2001). Briefly, cells were injected with LY by continuous current and stained with an anti-LY antibody (1:400 000, Sigma). Only cells identified as pyramidal neurons (recognized by the labeling of the apical dendrite proximal portion) and whose entire basal dendritic arbor was completely filled were included in the analysis. Pyramidal cortical cells were drawn with the aid of a camera lucida microscope attachment. Morphometric analysis was performed using NIH image software (NIH Research Services) (Ballesteros-Yânez et al. 2006). Size of dendritic arbors was determined by calculating the area contained within a polygon that joined the outermost distal tips of the basal dendrites (Elston and Rosa 1997, 1998). Branching pattern was determined by counting the number of dendritic branches that intersected concentric circles (centered on the cell body) of radii that incremented by $25 \mu \mathrm{m}$ (Sholl 
1953). Dendritic spines on labeled pyramidal cells were measured by counting the number of spines in each $10-\mu \mathrm{m}$ segment $(\times 100$ oil objective) from the soma to the distal tips of dendrites. Spines were counted on 10 horizontally projecting dendrites per animal, randomly taken from different cells. The estimation of the total number of spines in the basal dendritic tree was calculated by multiplying the average number of spines of a given portion of the dendrite by the average number of branches for the corresponding region over the entire dendritic tree.

\section{Transfection, Staining, and Morphometric Analysis of Primary Cortical Cultures}

Cortical primary cultures were prepared from 17.5-day-old embryos (E17.5) and maintained in Neurobasal B27-supplemented medium (Invitrogen). Cortical cultures were transfected with an EGFP plasmid by Lipofectamine 2000 according to manufacturer instructions. Cells at selected day-in-vitro (DIV) of interest were fixed with $4 \%$ PFA at room temperature for $20 \mathrm{~min}$ and subjected to immunofluorescence analysis with the indicated antibody according to standard protocols (antiPSD95 [1:700, ABCAM], antisynaptophysin [1:400, Sigma], anti-tubulin [1:1000, Upstate]). Actin was stained using rhodamine phalloidin (1:400, Sigma). For morphometric analysis, positive cells expressing EGFP were recorded in 3D image stacks with a ZEISS Axioplan2 fluorescence microscope and manually reconstructed with the ImagePro Plus 5 application Neurodraw. After reconstruction, we calculated surface area of the soma; number of dendrites per cell, total dendrite length, number of branch points, terminal segment length (the segment after the last branch point of the neurite), intermediate segment length, number and length of filopodia, and growth cones. Reconstruction of spines from mature cultures was performed with Imaris software, and the density of spines in $30 \mu \mathrm{m}$ of dendrite was measured. Spines were assigned to several morphological categories: type $\mathrm{A}$, thin spines without a clear differentiated head; type $B$, long neck with small round head; and type $\mathrm{C}$, short neck with round large head (Fig. $4 E$ ).

\section{Confocal Microscopy and Live Cell Imaging}

Fixed images of cortical cultures were acquired with a confocal Leica TCS SPE microscope and further analyzed with Image J software. For the analysis of growth cone area free of microtubule invasion, ratio between the areas occupied by F-actin versus tubulin of a projected image from stacks was analyzed. Synaptic density was calculated for each neuron as number of mature synaptic contacts (PSD95 and synaptophysin) per 100 microns of dendritic length and normalized by total cell density of its respective coverslip. For life cell imaging, phase contrast was used to record stage coordinates of suitable axonal growth cones with the aid of a Zeiss Cell Observer HS microscope coupled to a temperature-controlled chamber at $37^{\circ} \mathrm{C}$. Cultures were treated with harmine $(0.1 \mu \mathrm{M})$ to inhibit Dyrk1A activity. Images were acquired at DIV 1 every $5 \mathrm{~min}$ for a period of $14 \mathrm{~h}$ using $40-\mathrm{ms}$ integration time and analyzed with particle track plugging of Image J software.

\section{FRAP Analysis of Actin Dynamics in Dendritic Spines}

FRAP experiments were performed in DIV 22 cortical cultures transfected by YFP-actin DNA electroporation using the Mouse Nucleofector kit (Amaxa biosystems) following manufacturer's instructions. Photobleaching was carried out using a Leica TCS SL confocal microscope based on previous work (Star et al. 2002). The fluorescence recovery after photobleaching represents the incorporation of new YFP-actin monomers into the actin filament. The net fluorescence recovery at the steady-state (mobile fraction) represents the free diffusion of YFP-actin monomers and the proportion of filaments in dynamic equilibrium. Lower proportions of stable filaments render high values of mobile fraction and, conversely, a high proportion of stable actin filaments will produce smaller mobile fraction values. FRAP experiments were performed using the following protocol: 10 single prebleach scans were acquired at 356-ms intervals followed by 20 bleach scans at full power laser line using a circular area of $2.59 \mathrm{\mu m}^{2}$.
Mean fluorescence intensity during the time series was quantified using the Image Processing Leica Confocal Software.

\section{Electrophysiological Recordings}

Extracellular spontaneous activity of cortical primary cultures was recorded using E2 Dish device (Aleria Biodevices) constituted by a plastic culture substrate supporting low-complexity multiunit loosepatch recording of developing networks (Morales et al. 2008). Briefly, the system consists in an hybrid polydimethylsilane-on-polystyrene structure including 2 chambers $(6 \mathrm{~mm}$ in diameter) separated by a microchannel (1 $\mathrm{mm}$ in length) serving as substrate-embedded recording pipettes. Spikes up to $50 \mathrm{pA}$ in voltage-clamp and $300 \mathrm{mV}$ in current-clamp modes were recorded in sparse and bursting activity patterns with the aid of E2 soft software following manufacturer instructions.

\section{Immunoblotting}

Immunoblotting was performed following standard protocols in E17.5, postnatal P7 and P10, and adult cortical extracts and mouse cortical culture lysates ( 3 DIV). Blotts were incubated with primary anti-Tau (1:1000, Bioscience), anti-PSD95 (1:1000, ABCAM), anti-synaptophysin (1:1000, Sigma), or anti-GAPDH (1:1000, Chemicon) antibodies overnight at $4{ }^{\circ} \mathrm{C}$, followed by horseradish peroxidase-labeled antispecies-specific antibody. Quantification was performed by densitometric analysis (Image Gauge software). Blots were normalized with the levels of GAPDH.

\section{Quantitative Real-Time PCR}

For quantitative real-time polymerase chain reaction (qPCR) from DIV3 cultures, total cellular RNA was isolated from cortical cultures by using the RNeasy MiniKit (Quiagen). One microgram of total RNA was used for firststrand complementary deoxyribonucleic acid (CDNA) synthesis by using Omniscript Reverse Transcription kit (Quiagen). Gene expression was determined by SYBR Green QPCR, using SYBR Green PCR mix (Roche) CDNA as template. The following gene-specific primers were used: Tau_F: 5'-CTGAAGCACCAGCCAGGAGG-3', Tau_R: 5'-TGGTCTGTCTTGGCTTT GGC-3', L1cam_F: 5'-ACAGCGCATGGÄAGACTTGA-3', L1cam_R: 5' CGGGTGGTAAGAAAGAGACTCG-3', Elmo2 F: 5'-ATCCACCAGGAGTCATGAAGTCA-3', Elmo2_R: 5'-AAGCCCGACCCAGGCTC-3'. qPCR was performed on a LightCycler LC480 instrument (Roche), using the following amplification conditions: $5 \mathrm{~min}, 95^{\circ} \mathrm{C}$; followed by 45 cycles of $10 \mathrm{~s} ; 95^{\circ} \mathrm{C}, 30 \mathrm{~s} ; 62{ }^{\circ} \mathrm{C}, 15 \mathrm{~s} ; 72{ }^{\circ} \mathrm{C}$. Cp values were determined using the LightCycler $480 \mathrm{SW} 1.5$ software (Roche). The Pgk-1 (phosphoglycerate kinase 1) housekeeping gene was used to normalize gene expression.

\section{Statistical Analysis}

All data were normally distributed, thus allowing the use of parametric tests. For comparison between 2 groups, the Student's $t$ test was used. In the case of morphological parameters of cortical pyramidal neurons and synaptogenesis analysis, both experiments with more than 2 groups, two-way analysis of variance (ANOVA) with Bonferroni as post hoc test was performed. Repeated measure ANOVA was used to analyze data curves of Sholl analysis. All graphs were plotted as mean \pm standard error of the mean. All statistical analyses were performed using SPSS and GraphPad Prism 5 software packages.

\section{Results}

\section{Overexpression of Dyrk1A Reduces Dendritic Arborization Complexity in Adult Mice}

Layer II/III pyramidal cells from the M2 region of adult ( 2 months) TgDyrk1A and wild type littermates were injected with LY (66 and 96 cells, respectively) (Fig. 1A). The size of the basal dendritic field area of TgDyrk $1 \mathrm{~A}$ was similar to wild types (mean \pm standard deviation: $3.42 \pm 0.98 \times 10^{4} \mu \mathrm{m}^{2}$ and $3.38 \pm$ $0.91 \times 10^{4}{\mu \mathrm{m}^{2}}^{2}$, respectively). However, TgDyrk1A showed 
A

Wild type

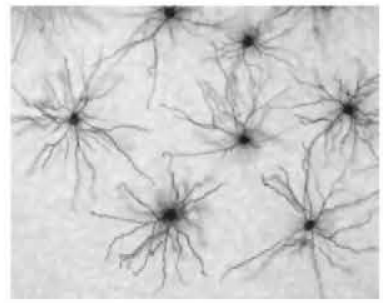

TgDyrk1A

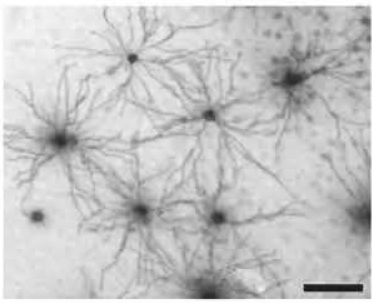

B

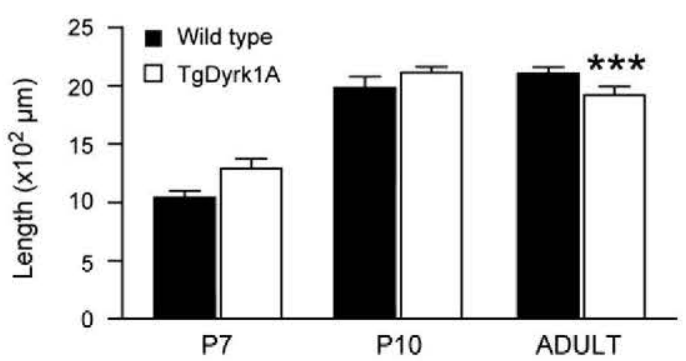

C

P7 sholl analysis

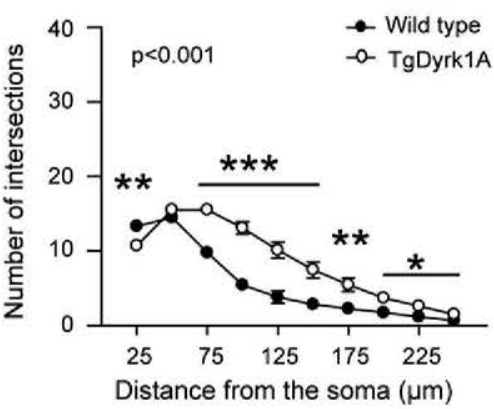

F

Spine density

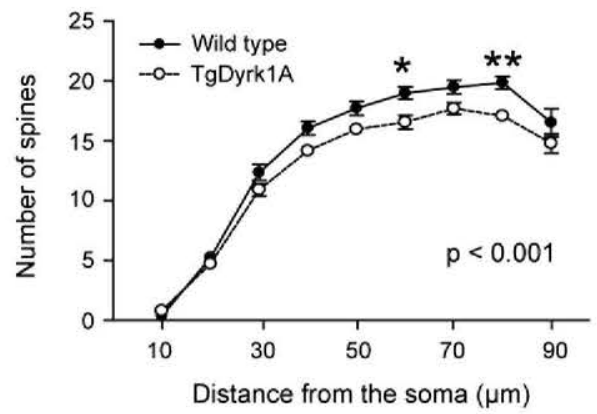

D

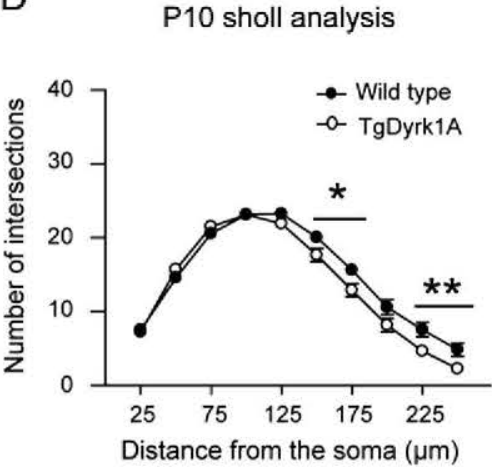

G

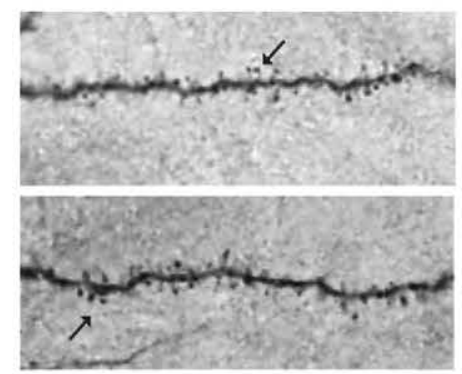

E

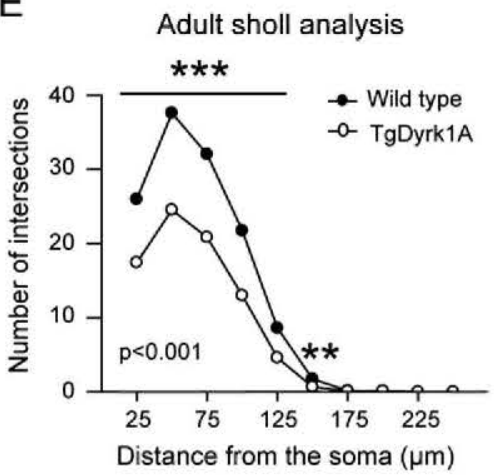

TgDyrk1A

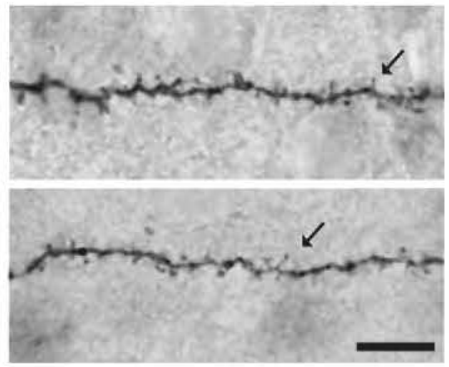

Figure 1. Dyrk1A overexpression recapitulates DS dendritic phenotype. Morphological characterization of the basal dendritic fields was performed in cortical neurons at P7, P10, and adult TgDyrk1A mice and their wild type littermates. (A) Representative photomicrographs of adult wild type (left panel) and TgDyrk1A mice (right panel) neurons from cortical layer III injected with LY. Scale bar $=125 \mu \mathrm{m}$. (B) Total length of basal dendrites. Sholl analysis (number of dendritic branches that intersected concentric circles [centered on the cell body] of radii that incremented by $25 \mu \mathrm{m}$ ) of $(C) P 7,(D) P 10$, and $(E)$ adult dendrites to determine the complexity of the dendritic trees. $(F)$ Density of spines along the entire length of the dendrites that were only quantified in adult mice. (G) Photomicrographs of horizontally projecting basal dendrites from adult wild type (left panels) and TgDyrk1A mice (right panels). Arrows show the spines along the neurite. Scale bar $=10 \mu \mathrm{m}$. Black bars and symbols represent wild type, and white bars and symbols represent TgDyrk1A mice. Data are represented as means \pm standard error of the mean; ${ }^{*} P<0.05,{ }^{* *} P<0.01,{ }^{* * *} P<0.001$.

significantly shorter dendrites $\left(1.90 \pm 0.51 \times 10^{3} \mu \mathrm{m}\right.$ vs. $2.31 \pm$ $0.50 \times 10^{3} \mu \mathrm{m}$, respectively, Fig. $\left.1 \mathrm{~B}\right)$. The quantification of dendritic branches as a function of the distance from the cell body (Fig. $1 E$ ) revealed that the peak branching complexity of TgDyrk1A arbors was less $(24.54 \pm 5.71$ intersections, Sholl analysis) than in wild types (37.60 \pm 6.82 intersections), although peak complexity was localized at the same distance (50 microns) from the soma in both genotypes. The analysis of the branching patterns of the whole dendritic arbor revealed significant genotype-dependent differences (repeated measures ANOVA, $P<0.001$ ), particularly in the region of the arbor nearer to the soma (Fig. $1 \mathrm{E}$ ). Finally, the density of spines along the entire length of the dendrites was reduced in TgDyrk1A
(Fig. $1 F, G$ ). Statistical analysis showed the differences to be significant at 60 and 80 microns from the soma (repeated measures ANOVA $P<0.001$, post hoc Bonferroni: $P<0.05$ and $P<0.01$, respectively). The estimation of the total number of spines in the basal dendritic arbor revealed that pyramidal cells in TgDyrk1A had a $40 \%$ reduction in number of spines in their basal dendritic arbors compared with wild types. Moreover, qualitative analysis revealed differences in TgDyrk1A spine morphology. Whereas in wild type dendrites mostly mushroom-like protrusions were observed, TgDyrk1A dendrites showed a higher proportion of thin and long spines (Fig. $1 G$ ). Further experiments will be necessary to analyze in detail the $3 \mathrm{D}$ dendritic spine morphology using high 
magnification confocal images instead of images from neurons labeled with DAB.

\section{Overexpression of Dyrk1A Modulates Dendritic Arborisation during Postnatal Development in TgDyrk1A} We analyzed pyramidal cells of TgDyrk1A and their wild type littermates at P7 (49 and 62 cells, respectively) and P10 (40 and 55 cells, respectively), in the same layer and cortical area as in adults. Basal dendritic field areas showed no differences at $\mathrm{P} 7$ and $\mathrm{P} 10$ in TgDyrk1A respect to wild types (P7: $2.76 \pm 1.14$ $\times 10^{4} \mu \mathrm{m}^{2}$ and $2.39 \pm 1.01 \times 10^{4} \mu \mathrm{m}^{2}$, respectively; P10: 3.31 $\pm 0.82 \times 10^{4} \mu \mathrm{m}^{2}$ and $2.93 \pm 0.91 \times 10^{4} \mu \mathrm{m}^{2}$, respectively; figure not shown). No differences in total dendritic length of basal arbors (Fig. $1 B$ ) were observed at either P7 $\left(1.45 \pm 0.65 \times 10^{3}\right.$ TgDyrk1A and $1.25 \pm 0.43 \times 10^{3} \mu \mathrm{m}$, wild types $)$ or P10 (2.11 \pm $0.31 \times 10^{3}$ TgDyrk1A and $1.99 \pm 0.55 \times 10^{3} \mu \mathrm{m}$, wild types). At $\mathrm{P} 7$, the dendritic arbor of TgDyrk1A neurons was significantly more complex than in wild type (repeated measures ANOVA, $P<0.001$, Fig. $1 C$ ). These differences disappeared at P10, when mice of both genotypes presented similar branching complexity and distribution (Fig. $1 D$ ). At $\mathrm{P} 7$ and $\mathrm{P} 10$, due to the difficulty to distinguish between spines and filopodia, spine analysis was not carried out.

\section{Dyrk1A Overexpression Impairs Neurite Elongation of Dissociated Cortical Neurons in Primary Cultures of TgDyrk1A Mice}

The changes observed in adult and developing pyramidal cell morphology in TgDyrk1A mice suggested the involvement of Dyrk1A in cortical neuronal outgrowth and branching. In this process, adequate neurite outgrowth is required to establish mature connections between neurons. We analyzed EGFPtransfected neurons ( 71 wild type and $68 \mathrm{TgDyrk} 1 \mathrm{~A}$ ) of cortical primary cultures at DIV 3, using Thy-1 promoter to mainly detect pyramidal neurons. A significant decrease in the number of dendrites emerging from the soma and reduced dendritic complexity (number of branch points per dendrite) $(P<0.05$, Fig. 2A) was observed in TgDyrk1A compared with wild type neurons $(P<0.05$, Fig. $2 A)$. In TgDyrk1A, dendritic filopodia were shorter $(P<0.001$, Fig. $2 D)$ with a trend toward reduction in the axons $(P=0.07$, Fig. $2 D)$.

Additionally, since early stage neurons of primary cultures are already polarized, it was possible to distinguish between dendrites and axons (Dotti et al. 1988). In our experiments, we detected a slight reduction in axon length in TgDyrk1A neurons $(P=0.07$, Fig. $2 B)$. This reduction reached statistical significance in the terminal $(P<0.01$, Fig. $2 C)$ but not in the intermediate segments. No differences between both genotypes were observed in axon branching. However, TgDyrk1A axons presented fewer growth cones, main structure responsible for the elongation, and directionalization of the branches $(P<0.05$, Fig. $2 C)$.

\section{Overexpression of Dyrk1A Affects Axonal Growth by Modifying Cytoskeletal Machinery}

The reduced axonal length and numbers of axonal cones in TgDyrk1A neurons suggested that axonal growth might be compromised. While growing, axons exhibit a variety of dynamic behaviors; they often elongate in a saltatory manner, with periods of abrupt advance alternating with periods of retraction or pausing. We registered the axonal movements at the initial stages of primary cultures (DIV 1, 46 wild type and 40 TgDyrk1A neurons) using $14 \mathrm{~h}$ time-lapse recording (Fig. 3A). The axonal behavior was different in transgenic neurons, which showed a significant reduction of the distance traveled by the axon $(P<0.05$, Fig. $3 A, B)$. This phenotype was reverted after treatment with harmine, a potent Dyrk1A inhibitor (Gockler et al. 2009) $(P<0.01$, Fig. $3 B)$ at a dose not affecting neuronal viability in an MTT assay (data not shown). Speed of both forward and backward axon movements was reduced in TgDyrk1A axons $(P<0.005$ for both, Fig. 3C), with no genotype-dependent differences in the number of growth-cone directionality changes.

Since the rate of growth cone advance depends on the interaction between the F-actin cytoskeleton and microtubules, we double stained cortical neurons with a tubulin antibody and rhodamine phalloidin to label microtubule and actin filaments, respectively (DIV 1, 52 wild type and 49 TgDyrk1A growth cones). In TgDyrk1A neurons, tubulin staining was restricted neurite shaft, with significant less occupancy of the growth cone $(P<0.001$, Fig. $3 D, E)$, suggesting changes of microtubule distribution in transgenic neurons. The altered cytoskeletal distribution was detected in $64.63 \%$ of TgDyrk1A neurons compared with $19.85 \%$ of wild type neurons, transgenic neurons showing increased penetrance of the phenotype $(P<0.001)$.

Axonal elongation depends on stabilization of microtubule array, a process regulated by several microtubule associated proteins such as Tau. In immunoblotting analyses, Tau expression was reduced in both protein extracts from DIV3 transgenic cultures $(P<0.05$, Fig. $3 F)$ and in E17.5 embryo cortex $(P<0.05$, Fig. $3 F)$. In contrast, no significant differences were detected at adult and postnatal stages (data not shown). When Tau mRNA level in DIV3 cultures was analyzed no difference between genotypes was found (Fig. $3 G$ ).

\section{Dyrk1A Overexpression Influences Synaptogenesis}

In our experiments, Dyrk1A overexpressing neurons showed less branched neurites with shorter filopodia (see above). Since these alterations could affect the correct establishment of synaptic connections, we analyzed the number of putative synaptic contacts. Neurons at DIV 10, 15, and 20 were immunostained with a presynaptic and a postsynaptic marker being their co-localization along the dendrites defined as a synapse (Fig. $4 \mathrm{~A}$ ). Although at DIV 10 no differences were observed between genotypes, synaptic density was higher in TgDyrk1A dendrites at DIV $15(P<0.05$, Fig. $4 B)$. However, at more mature stages, a significant decrease in the synaptic density was noticed in TgDyrk1A, compared with wild type dendrites $(P$ $<0.001$, Fig. $4 B$ ), indicating an impaired mature synaptic contact formation during network establishment. Thus, the expected progressive increase of synaptic density was observed in wild type but not in TgDyrk1A dendrites (Fig. $4 B$ ). The density of dendritic spine in TgDyrk1A EGFP-transfected mature neurons was reduced $(P<0.001$, Fig. $4 C, D)$. Moreover, TgDyrk1A spines morphology differed from that in wild type ones. TgDyrk1A spines were mostly thinner and longer (spine type A, $P<0.001$ ), whereas wild types were characterized by big head with a short neck (spine type C, $P<0.001$ ) (Fig. $4 E, F$ ).

In cortical protein extracts from postnatal and adult mice (Fig. $4 G$ ) presynaptic synaptophysin and postsynaptic PSD95 were reduced in TgDyrk1A, at $\mathrm{P} 7(P<0.05$ and $P<0.01$, respectively) but not $\mathrm{P} 10$ or adult cortex. 

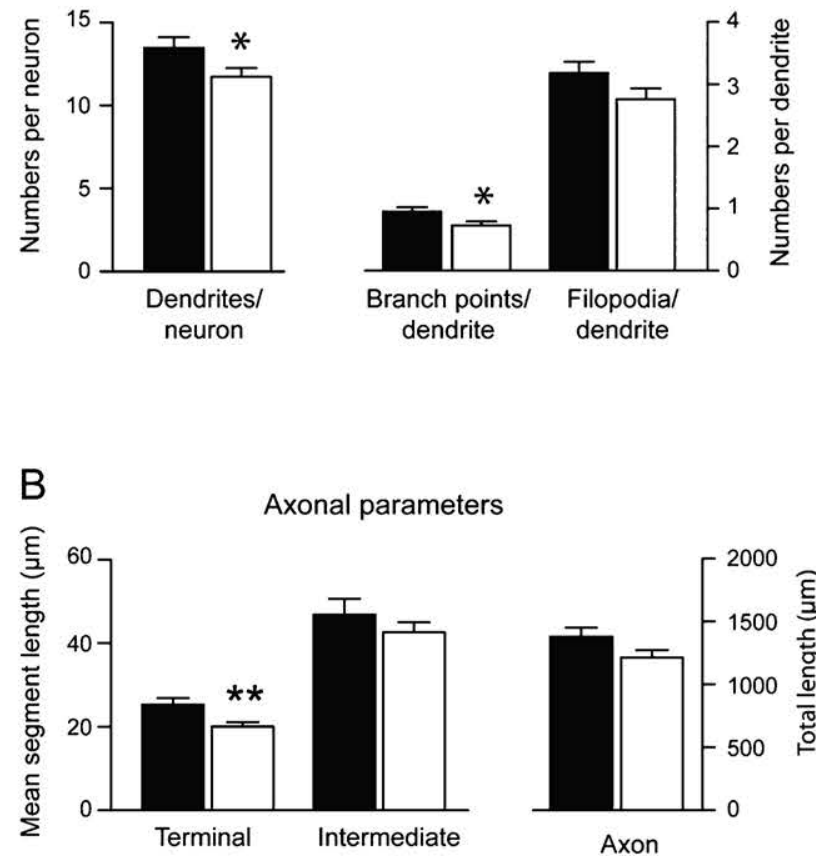

C Growth cones/axon

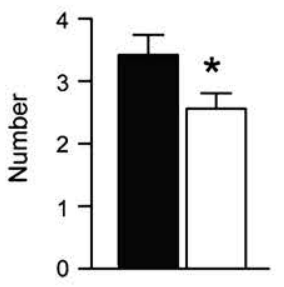

Wild type

$\square$ TgDyrk1A

D

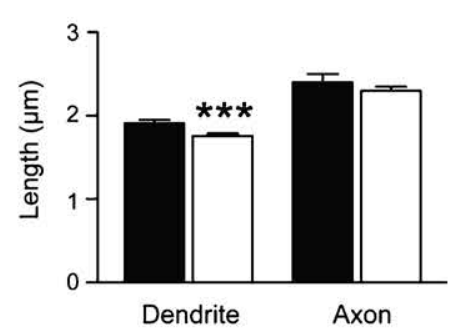

Figure 2. Neuritogenesis is altered in cultured cortical TgDyrk1A neurons. The reconstruction of cultured cortical EGFP-positive cells at DIV 3 allowed the analysis of the neuritogenesis at initial stages. Histograms represent: $(A)$ the dendritic tree complexity, as measured by the number of dendrites emerging from soma, the number of branch points and the number of filopodia per dendrite; $(B)$ the mean intermediate and terminal segment length and total length of the axon; $(C)$ the total number of growth cones per axon; (D) mean filopodia length in dendrites and axons. Black bars are wild type, and white bars, TgDyrk1A cultures. Data are represented as means \pm standard error of the mean. ${ }^{*} P<0.05,{ }^{* *} P<0.01,{ }^{*}{ }^{*} P<0.001$.

\section{Dyrk1A Overexpression Induces Alterations in Actin Dynamics in Dendritic Spines}

Spines are unstable and motile structures submitted to constant morphological changes that rely on actin cytoskeleton rearrangements, where diffusion of actin monomers between the dendrite and the spine allows their incorporation into actin filaments to establish the spine actin network. We evaluated actin dynamics in spines using FRAP in YFP-actin transfected mature cortical cultures (DIV 22) (Fig. 5A). No relation between YFP expression level and the percentage of mobile actin fraction in either genotype was found, indicating that overexpression of YFP-actin did not alter the dynamics of actin (Fig. 5B). Mobile actin showed a different behavior in both genotypes (representative actin dynamics curves shown in Fig. 5C). In average, the mobile fraction was lower in TgDyrk1A spines $P<0.05$, Fig. $5 D$ ), indicating a higher fraction of stable filaments (Fig. $5 D$ ). Also, the fluorescence recovery half time was faster in transgenic spines $(P=0.05$, Fig. $5 D)$, suggesting a modified actin treadmilling. These disturbances in actin dynamics and proportion of stable filaments could affect synaptic plasticity events in TgDyrk1A.

\section{Altered Neuronal Activity in TgDyrk1A Cortical Cultures}

Since the reduced synaptic density observed in TgDyrK1A mice would influence the synaptic connectivity established in culture, we have examined the spontaneous electrical activity of both wild types and TgDyrK1A culture neurons. To this end, an extracellular electrophysiological recording (50 wild type and 46 TgDyrk1 A cultures) of $5 \mathrm{~min}$ in length were analyzed for firing frequency using E2-Dish apparatus (Fig. 6A). TgDyrk1A cultures showed a significantly reduced number of spikes along the recording time $(P<0.05$, Fig. $6 B, C)$, indicating an altered firing consequent with the altered synaptogenesis and reduced axonal developing.

\section{Discussion}

We here demonstrate that Dyrk1A overexpression in TgDyrk1A leads to morphological alterations in pyramidal dendritic trees. This phenotype is suggested to depend on abnormal microtubule distribution during neuritogenesis and actin dynamics during synaptogenesis, affecting neurite and dendritic spine development.

\section{In Vivo Dyrk1A Overexpression Alters the Microarchitecture of Cortical Pyramidal Neurons}

DS patients show alterations in cortical dendritic trees, a microanatomical trait that has been proposed as determinant of the deficient information processing in mental retardation (Ramakers 2002; Dierssen and Ramakers 2006). We here present evidence that DYRK1A overexpression produces a dendritic phenotype similar to that observed in Ts65Dn mice (Dierssen et al. 2003) and DS brains (Takashima et al. 1981; Becker et al. 1991). Adult TgDyrk1A show reduced cortical pyramidal complexity, spine density and dendritic branching, and length. Interestingly, Dyrk1A heterozygous mice (Fotaki et al. 2002) also show alterations in cortical neuron cytoarchitecture characterized by a reduction in dendritic length and complexity, suggesting that subtle dosage variations of Dyrk $1 \mathrm{~A}$ can lead to alteration in pyramidal cortical neurons.

A relevant role of DYRK1A has been previously reported during corticogenesis (Rahmani et al. 1998; Marti et al. 2003; Hammerle et al. 2008). The morphological study of cortical pyramidal cells of TgDyrk1A mice at postnatal ages, when experiencedependent maturation of the dendritic tree takes place, showed 

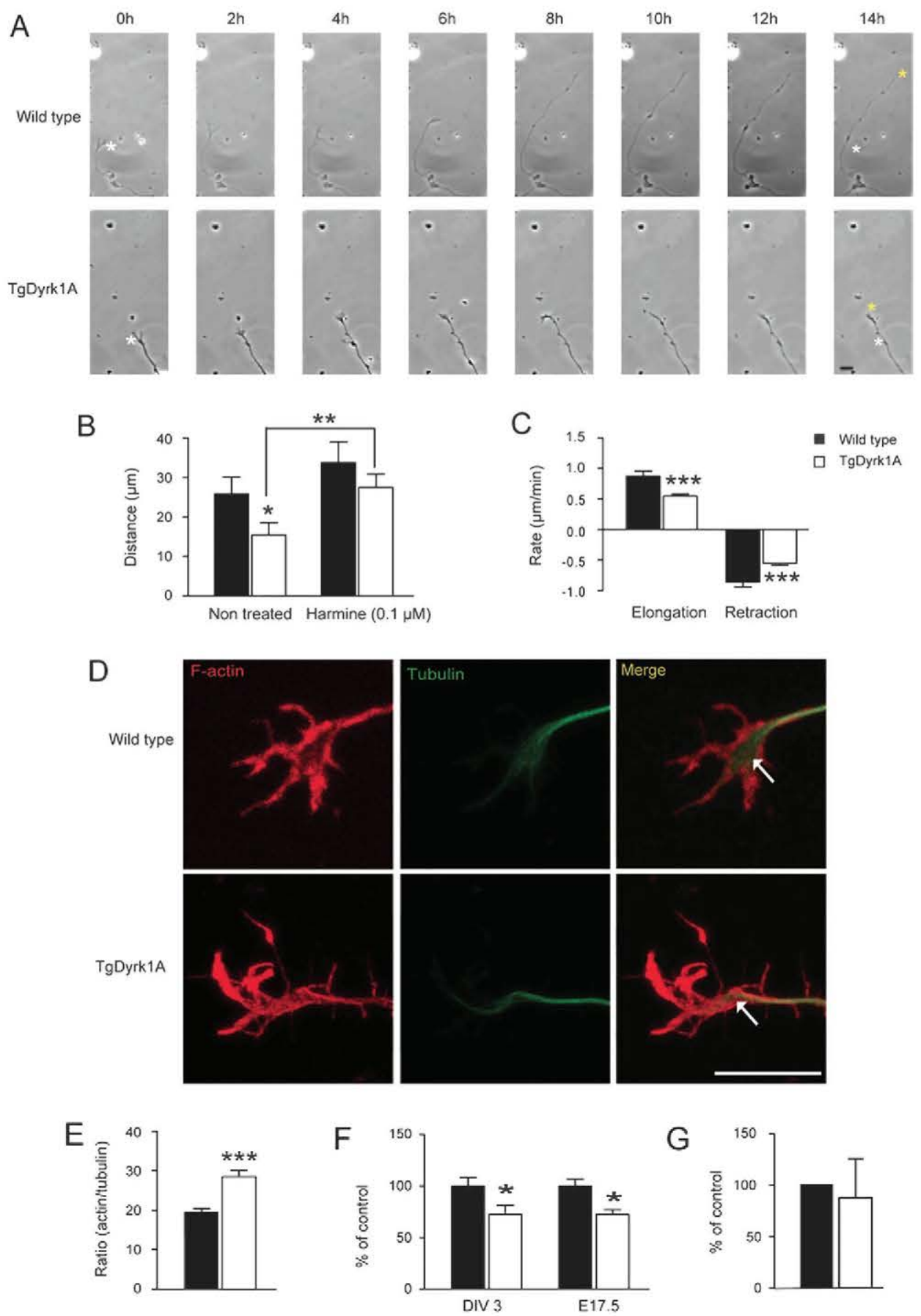

Figure 3. TgDyrk1A neurons showed impaired axon elongation associated with changes in microtubule cytoskeleton. Phase contrast time-lapse experiments for 14 h of DIV 1 primary cortical culture were performed to analyze axon growth. (A) Representative images of axonal outgrowth, being white asterisk the initial position and yellow one the final reach point of the growth cone, indicating final traveled distance. The histograms represent the quantification of $(B)$ final distance traveled by the axon and $(C)$ mean speed of forward and reward axon movements. (D) Representative confocal images of immunolabelled growth cones at DIV 1 with F-actin (red), $\alpha$-tubulin (green), and co-localization of both. Arrows show the extension of microtubules along the neurite and their interaction point with the actin. Upper panel: wild type, lower panel: TgDyrk1A. (E) Histogram represents the ratio between the area occupied by actin with respect to tubulin. (F) Densitometric quantification of immunoblotting graphed as percentage respect to wild type values of total Tau in DIV3 cultures and E17.5 embryo cortex. (G) Histogram represents the ratio of R3-Tau mRNA level respect to wild type values obtained by qPCR. Black bars = wild type, white bars = TgDyrk1A. DIV: day in vitro. Data are represented as the means \pm standard error of the mean. ${ }^{*} P<0.05,{ }^{* *} P<0.01,{ }^{* * *} P<0.001$. Scale bar $=10 \mu \mathrm{m}$.

that at early stages (P7), dendritic trees were more complex with longer branches. By P10, these differences had disappeared, while adult dendritic trees showed reduced morphological complexity. These findings recapitulate the changes observed in DS newborn and children (Becker et al. 1986; Prinz et al. 1997), where normal or even increased dendritic branching is observed
(Takashima et al. 1994; Vuksic et al. 2002) that contrasts with the reduced number of dendrites observed in later stages (Becker et al. 1991). This is the first time that the progression of postnatal regressive cortical changes of DS has been recapitulated in a mouse model and that a specific gene can be directly associated with the progression of the phenotype. 
A
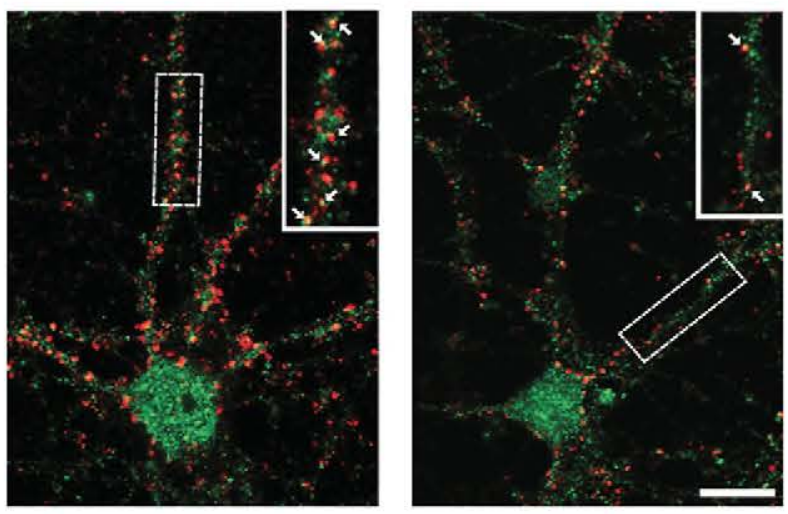

C
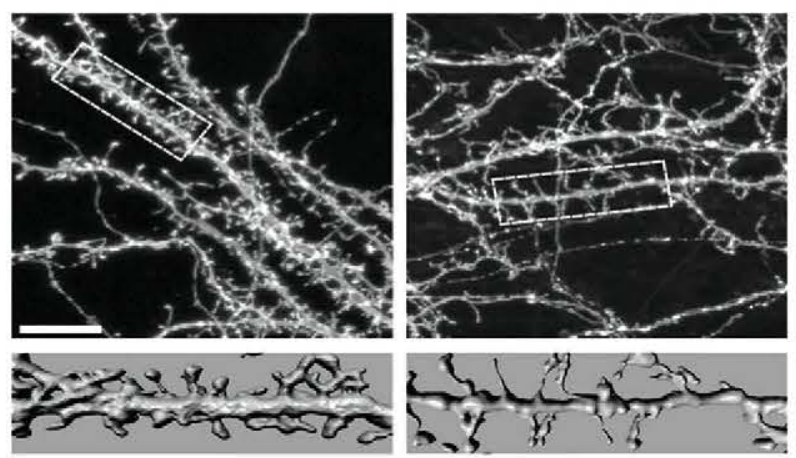

$E$

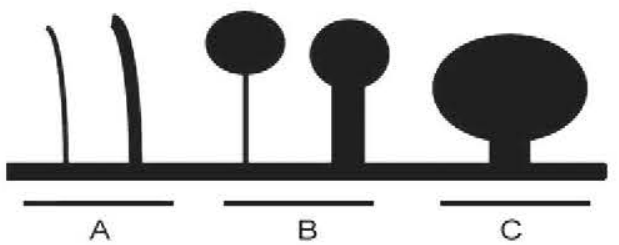

B

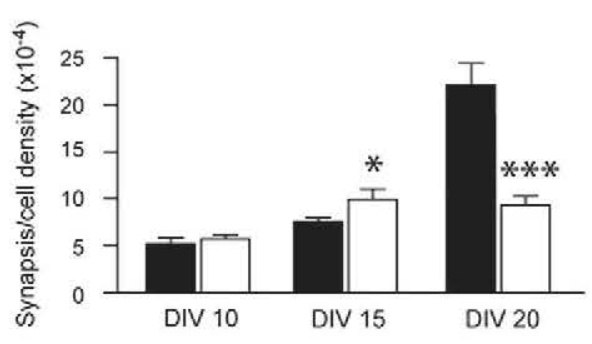

Wild type

D

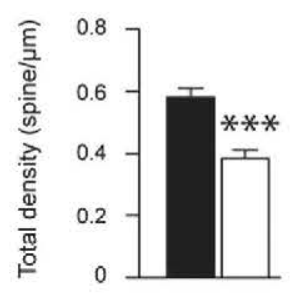

F

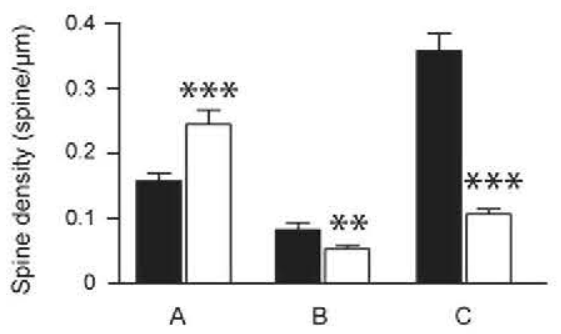

G

\begin{tabular}{|c|c|c|c|c|c|c|c|c|c|}
\hline & \multicolumn{3}{|c|}{ P7 } & \multicolumn{3}{|c|}{ P10 } & \multicolumn{3}{|c|}{ Adult } \\
\hline & Wild type & TgDyrk1A & $\mathrm{p}$ & Wild type & TgDyrk1A & $p$ & Wild type & TgDyrk1A & $p$ \\
\hline PSD95 & $100 \pm 10.7$ & $43.8 \pm 7.3$ & $\star *$ & $100 \pm 4.0$ & $93.7 \pm 7.1$ & n.s. & $100 \pm 24.8$ & $55.2 \pm 6.9$ & n.s. \\
\hline SY38 & $100 \pm 11.6$ & $60.9 \pm 11.6$ & * & $100 \pm 11.1$ & $76.3 \pm 3.2$ & $p=0.1$ & $100 \pm 13.4$ & $68.0 \pm 6.9$ & $p=0.08$ \\
\hline
\end{tabular}

Figure 4. Synaptogenesis is altered by Dyrk1A overexpression. (A) Representative confocal images of neurons at DIV 20 immunostained against presynaptic synaptophysin (red) and postsynaptic PSD95 (green) considering the co-localization of both a mature synaptic contact. Right upper insert corresponds to a higher magnification of the dendrite boxed. Arrows denote co-localization. (B) Histogram represents the synaptic density normalized by total cell density at DIV 10, 15, and 20. (C) Representative confocal images showing EGFP-transfected dendrites with spines. Lower surface reconstruction corresponds to a higher magnification of the dendrite boxed of 20- $\mu \mathrm{m}$ length. (D) Histogram represents the total dendritic spine density. $(E)$ Illustration of the morphological categories used to classity spines and $(F)$ density of each type of spine. $(G)$ Table showing the percentage respect to wild type values of western blot densitometric quantification of synaptophysin (Sy38) and PSD95 from cerebral cortex of postnatal days P7 and P10 and adult mice. Black bars are wild type and white bars are TgDyrk1A mice. DIV: day in vitro. Data are represented as means \pm standard error of the mean. ${ }^{*} P<0.05,{ }^{* *} P<0.01,{ }^{* * *} P<0.001$. n.s. non significant. Scale bar $=10 \mu \mathrm{m}$.

\section{Dyrk1A Participates in Neuritogenesis by Modifying the Cytoskeleton}

To get insight into the possible mechanisms, we used primary cortical cultures. Cultured TgDyrk1A neurons presented reduced axonal length, with shorter terminal segments and less complex dendritic arbors with fewer dendrites, branch points, and terminal segments. These results support and extend previous findings that suggested a role of DYRK $1 \mathrm{~A}$ on neuritogenesis. In an immortalized hippocampal progenitor cell line, overexpression of kinase-deficient DYRK1A impeded neurite outgrowth (Yang et al. 2001), and recently, a severe reduction of dendritic growth and complexity was reported after single Dyrk1A overexpression with adenovirus vectors in primary mouse cortical cultures and in dissociated cortical 

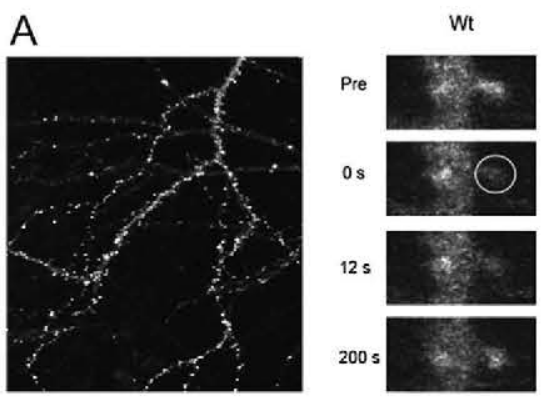

TgDyrK1A

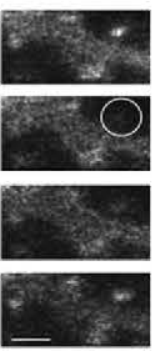

C

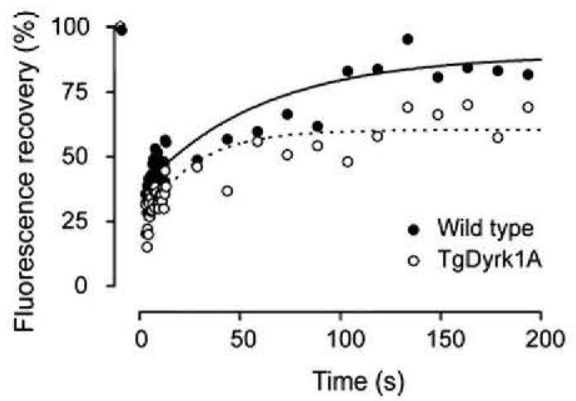

B

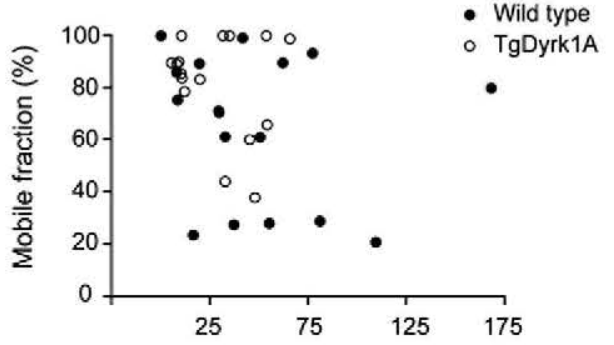

Spine initial intensity (a.u.)

D

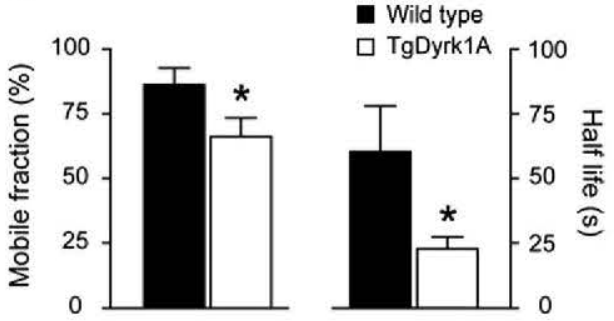

Figure 5. Actin dynamics was altered in spines of TgDyrk1A neurons. (A) Left panel: representative photomicrograph of a DIV 22 neuron expressing YFP-actin. YFP-actin was highly concentrated in dendritic spines, allowing an easy identification. Right panel: representative examples of wild type and TgDyrk1A spines at sequential fluorescence recovery stages after photobleaching. Initially, both spines showed fluorescence (Pre $=$ prebleaching) that disappeared after photobleaching (time $=0 \mathrm{~s}$ ). (B) Representation of the percentage of actin mobile fraction versus spine initial fluorescence, normalized in each spine respect to its background. No correlation was found between both parameters in each genotype (a.u. arbitrary units). (C) Illustrative example of actin dynamics in a wild type and a TgDyrk1A spine. (D) Histograms represent the mean average data of actin mobile fraction and half-time recovery of the fluorescence. Black circles and bars represent wild type mice; white circles and bars are TgDyrk1A. Data are represented as the means \pm standard error of the mean. ${ }^{*} P<0.05$. Scale bar $=2 \mu \mathrm{m}$.

A

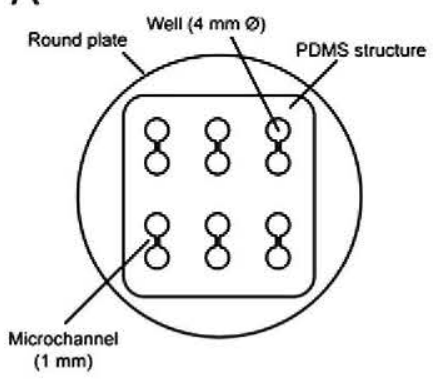

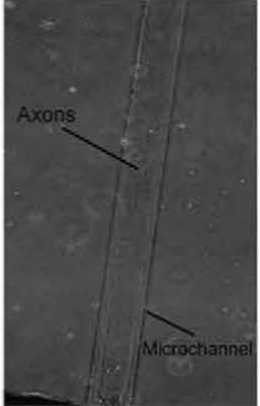

B

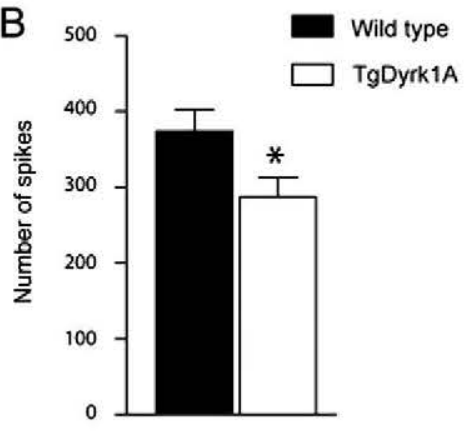

C

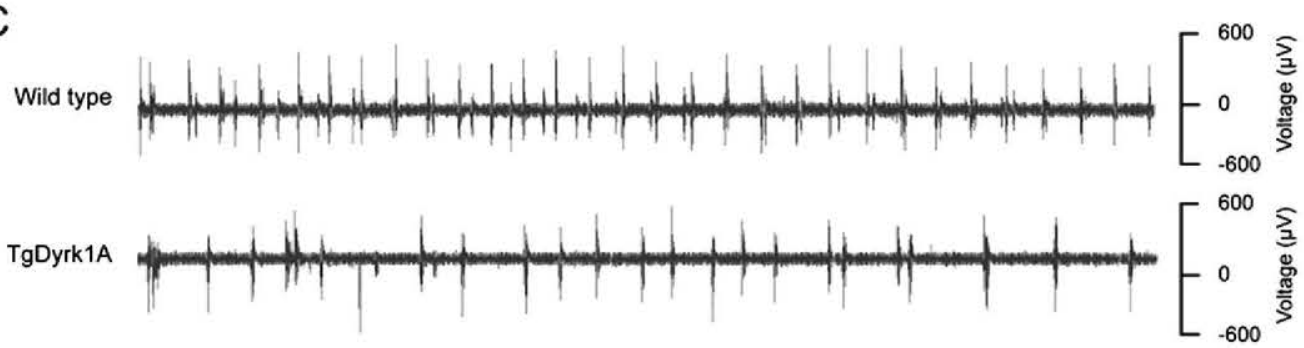

Figure 6. Spontaneous activity was reduced in TgDyrk1A neurons. (A) Schematic illustration of E2-Dish system where cultures were seeded to obtain electrophysiological recordings. The photomicrograph shows axons coming from both wells growing along the microchannel (1-mm length). (B) Histogram represents the number of spikes fired during $5 \mathrm{~min} .(C)$ Representative electrophysiological recordings of the spontaneous activity registered in both genotypes. Black bars are wild type and white bars are TgDyrk1A cultures. Data are represented as means \pm standard error of the mean. ${ }^{*} P<0.05$.

neurons from 152F7 YAC transgenic mice overexpressing Dyrk1A (Lepagnol-Bestel et al. 2009). These authors propose that Dyrk1A interact with the REST/NRSF chromatin-remodeling complex to deregulate genes such as Elmo2 or L1cam related to neurite outgrowth. However, no expression changes of neither of those proteins were found in our cortical cultures (data not 
shown), probably due to a temporal regulation of those genes (E12.5 vs. E17.5 in our case) or methodological artifacts (E12.5 telencephalon comparing with cortical cultures at DIV3). However, our results do not discard that Dyrk1A could be interacting with other protein involved in neuritogenesis. In fact, our previous studies (Toiber et al. 2010) also found DS-like brain splicing machinery malfunctioning in Dyrk1A overexpressing mice. Specifically, in both fetal DS brains and TgDyrk1A, we found modification in splicing-associated transcripts involved in synaptic functions. Among those, reduced levels of the dominant-negative TRKBT1 transcript, but not other TRKB mRNA transcripts, were accompanied by corresponding decreases in BDNF, a protein crucial for neuritogenesis. Moreover, here we demonstrated that in vivo overexpression of Dyrk1A alone is sufficient to induce a dendritic but also an axonal phenotype, opening avenues to therapy.

We then analyzed the dynamic properties of axonal growth in time-lapse experiments. The final distance traveled by TgDyrk1A neurites was shorter than that reached by wild type axons due to slower axonal growth cones movements and less prolonged rescue periods. This is probably due to the fact that Dyrk1A overexpression affected the distribution of microtubules respect to actin filaments as shown in TgDyrk1A growth cones, since the interaction of these cytoskeletal elements is crucial for the correct elongation of the axon.

Previous studies have demonstrated that Dyrk1A primes GSK3 $\beta$ phosphorylation of MAP1B, a phosphoprotein that regulates microtubule dynamics in growing axons, altering microtubule stability, and therefore axonal outgrowth (Scales et al. 2009). However, the discrepancy of the results obtained from COS-7 cell line and primary cortical cultures in their work suggest that other Dyrk1A substrates than MAP1B such as Tau (Kimura et al. 2007; Ryoo et al. 2007) could be contributing to microtubule dynamic. For this reason, we have analyzed Tau expression in our TgDyrk1A cultures. TgDyrk1A cultures presented reduced Tau protein levels similarly as occurs at late embryonic stage. Decreased expression of Tau could reduce the motility of the growth cone and inhibit axon outgrowth (Liu et al. 1999; Caceres et al. 2001; Dawson et al. 2001). The fact that no statistical differences were found in adult and postnatal ages, suggests that low Tau levels adjust during development matching with the peak of Dyrk1A expression. The reduction in Tau protein expression could be caused by a deregulation of gene expression or posttranslational modifications. Our qPCR experiments did not revealed changes in gene expression of $3 R$ isoforms, the most common in fetal brain, but as Dyrk1A has been widely associated with Tau alternative splicing it cannot be discard that other of the multiple Tau isoforms could be contributing to the observed phenotype. Thus, further experiments to confirm those points would be necessary. We propose that abnormal regulation of Tau in DS may affect the cortical neuronal network formation in developmental phases, promoting disintegration of the network in older adults. However, further experiments are necessary to verify this hypothesis.

\section{Dyrk1A Modulates Synaptogenesis and Modifies Spine Actin Dynamics}

Opposite to wild type cultured neurons, which showed a progressive increase of mature contacts along time, synaptogenesis did not further progress from DIV 15 onward in transgenic neurons. This resulted in a dramatic reduction of the number of synapses in the mature transgenic culture (DIV 20), indicating that Dyrk1A overexpression is an important factor in synaptic connectivity. In fact, its overexpression has been previously involved in plasticity of mature synapses in adult mice with enhanced hippocampal long-term depression (LTD) and reduced long-term potentiation (Ahn et al. 2006), suggesting a putative role in the regulation of synaptic and structural plasticity in the adult. Furthermore, there is a reduced expression of synaptic markers in TgDyrk1A cortex at $\mathrm{P} 7$, similar to the observations in trisomic Ts65Dn mice at comparable postnatal ages (Chakrabarti et al. 2007; Pollonini et al. 2008). Interestingly, at this stage, increased complexity of the dendritic arbor is observed. One of the main processes during postnatal development consists in the establishment of neuronal networks trough the creation of stable synaptic contacts. The inability to perform those contacts involves the retraction of emerging structures such as filopodia, driving to a reduction in branching and synaptogenesis. In our model, although there was increased branching at $\mathrm{P} 7$, the reduction in the expression of presynaptic and postsynaptic protein suggest a deficit in the establishment of new synaptic contact with consequences in later stages in branching. On the other hand, since defects in pruning leading to increased filopodia have been reported in other intellectual disability associated syndromes, this could also be an explanation of the observed phenotype.

The dendritic spines are the receiving parts of excitatory synapses and can dynamically change shape in response to memory-inducing stimuli. In mental retardation, the main morphological alteration affects dendritic spine shape (Kaufmann and Moser 2000; Ramakers 2002). Cultured TgDyrk1A neurons present shorter filopodia-like instable spines, with small heads and long necks and mature transgenic cultures show spine dysgenesia, a phenotype also detected in the preliminary observations from adult cortical spines. The morphology of dendritic spines is determined by the actin cytoskeleton, which is highly concentrated in dendritic spines (Matus 2000). TgDyrk1A spines present a significant reduction of the percentage of actin mobile fraction, suggesting a lower rate of treadmilling of actin filaments. This behavior may partly be related to altered actin polymerization, since a similar phenotype was previously described in wild type primary cultures treated with cytochalasin $\mathrm{D}$, which prevents actin polymerization (Star et al. 2002).

In our experiments, while the majority of the actin is in a dynamic status in control spines, TgDyrk1A spines showed a lower proportion of mobile actin, suggesting that the ability for plastic changes is compromised. On the other hand, the decreased fluorescence recovery time in TgDyrk1A spines reflected a faster actin filament turnover. It is important to remark that the actin turnover is independent of the synapse size (Star et al. 2002), thus TgDyrk1A disgenesis might not affect. A decrease in the fluorescence recovery was reported upon physiological stimuli leading to LTD. Those stabilize a large fraction of spine actin, presumably in the filamentous form, indicating that actin filament turnover also relies on synaptic activity (Star et al. 2002). In TgDyrk1A cultures, an altered firing pattern was observed in transgenic neurons along with altered actin turnover. However, we cannot discard that the impaired neuronal activity could be also associated with changes in the proportion of NMDA subunits in our TgDyrk1A 
mice (Altafaj et al. 2008). Taken together, these results suggest that Dyrk1A might be involved in the regulation of cytoskeleton dynamics at the spine level, affecting both spine structure and function

We conclude that Dyrk1A plays a key role in the neuronal morphogenesis through regulation of cytoskeletal dynamics being a critical factor in the dendritic pathology associated with DS. Further studies using Ts65Dn cortical cultures are necessary to establish the relevance of those findings in a trisomic context. Our results suggest that pharmacological interventions addressed to change DS dendritic pathology might be considered as a suitable option for treatment.

\section{Funding}

Spanish Ministry of Science and Innovation (FCT-08-0782, SAF2007-60827, SAF2007-31093-E, SAF2010-16427 and Juan de la Cierva to I.B.-Y.); Spanish Ministry of Health (PI 082038), Marató TV3, Jerome Lejeune (JMLM/AC/08-044), Reina Sofia and Areces Foundations (to M.M., M.D. and M.C.); Cognition Program of the Netherlands Organization for Scientific Research (NWO 051.04.090 to G.R.) and EU (LSHG-CT-2006-037627; PSO9/ 02673; CureFXS ERA-NET E-Rare. EU/FIS PS09102673). The laboratories of M.D. and C.F. were supported by DURSI (09 2009SGR1313); The CIBER of Enfermedades Raras is an initiative of the ISCIII. The laboratory of J. DeF. was supported by CIBERNED (CB06/05/0066), Fundación CIEN (2008) and the Spanish Ministry of Science and Innovation (SAF2009-09394).

\section{Notes}

Conflict of Interest : None declared.

Ahn KJ, Jeong HK, Choi HS, Ryoo SR, Kim YJ, Goo JS, Choi SY, Han JS, Ha I, Song WJ. 2006. DYRK1A BAC transgenic mice show altered synaptic plasticity with learning and memory defects. Neurobiol Dis. 22:463-472.

Altafaj X, Dierssen M, Baamonde C, Marti E, Visa J, Guimera J, Oset M, Gonzalez JR, Florez J, Fillat C, et al. 2001. Neurodevelopmental delay, motor abnormalities and cognitive deficits in transgenic mice overexpressing Dyrk1A (minibrain), a murine model of Down's syndrome. Hum Mol Genet. 10:1915-1923.

Altafaj X, Ortiz-Abalia J, Fernández M, Potier MC, Laffaire J, Andreu N, Dierssen M, González-García C, Ceña V, Martí E, et al. 2008. Increased NR2A expression and prolonged decay of NMDA-induced calcium transient in cerebellum of TgDyrk1A mice, a mouse model of Down syndrome. Neurobiol Dis. 32:377-384.

Ballesteros-Yáñez I, Benavides-Piccione R, Elston GN, Yuste R, DeFelipe J. 2006. Density and morphology of dendritic spines in mouse neocortex. Neuroscience. 138:403-409.

Becker L, Mito T, Takashima S, Onodera K. 1991. Growth and development of the brain in Down syndrome. Prog Clin Biol Res. 373:133-152.

Becker LE, Armstrong DL, Chan F. 1986. Dendritic atrophy in children with Down's syndrome. Ann Neurol. 20:520-526.

Benavides-Piccione R, Dierssen M, Ballesteros-Yanez I, Martinez de Lagran M, Arbones ML, Fotaki V, DeFelipe J, Elston GN. 2005. Alterations in the phenotype of neocortical pyramidal cells in the Dyrk1A+/- mouse. Neurobiol Dis. 20:115-122.

Caceres A, Potrebic S, Kosik KS. 2001. The effect of tau antisense oligonucleotides on neurite formation of cultured cerebellar macroneurons. J Neurosc. 11:1515-1523.

Chakrabarti L, Galdzicki Z, Haydar TF. 2007. Defects in embryonic neurogenesis and initial synapse formation in the forebrain of the Ts65Dn mouse model of Down syndrome. J Neurosci. 27:11483-11495.
Dawson HN, Ferreira A, Eyster MV, Ghoshal N, Binder LI, Vitek MP. 2001. Inhibition of neuronal maturation in primary hippocampal neurons from tau deficient mice. J Cell Sci. 114:1179-1187.

Dierssen M, Benavides-Piccione R, Martinez-Cue C, Estivill X, Florez J, Elston GN, DeFelipe J. 2003. Alterations of neocortical pyramidal cell phenotype in the Ts65Dn mouse model of Down syndrome: effects of environmental enrichment. Cereb Cortex. 13:758-764.

Dierssen M, Herault Y, Estivill X. 2009. Aneuploidy: from a physiological mechanism of variance to Down syndrome. Physiol Rev. 89:887-920.

Dierssen M, Martinez de Lagran M. 2006. DYRK1A (dual-specificity tyrosine-phosphorylated and -regulated kinase 1A): a gene with dosage effect during development and neurogenesis. ScientificWorldJournal. 6:1911-1922.

Dierssen M, Ramakers GJ. 2006. Dendritic pathology in mental retardation: from molecular genetics to neurobiology. Genes Brain Behav. 5(Suppl 2):48-60.

Dotti CG, Sullivan CA, Banker GA. 1988. The establishment of polarity by hippocampal neurons in culture. J Neurosci. 8:1454-1468.

Elston GN, Benavides-Piccione R, DeFelipe J. 2001. The pyramidal cell in cognition: a comparative study in human and monkey. J Neurosci. 21:RC163.

Elston GN, Rosa MG. 1997. The occipitoparietal pathway of the macaque monkey: comparison of pyramidal cell morphology in layer III of functionally related cortical visual areas. Cereb Cortex. $7: 432-452$.

Elston GN, Rosa MG. 1998. Morphological variation of layer III pyramidal neurones in the occipitotemporal pathway of the macaque monkey visual cortex. Cereb Cortex. 8:278-294.

Fotaki V, Dierssen M, Alcantara S, Martinez S, Marti E, Casas C, Visa J, Soriano E, Estivill X, Arbones ML. 2002. Dyrk1A haploinsufficiency affects viability and causes developmental delay and abnormal brain morphology in mice. Mol Cell Biol. 22:6636-6647.

Gockler N, Jofre G, Papadopoulos C, Soppa U, Tejedor FJ, Becker W. 2009. Harmine specifically inhibits protein kinase DYRK1A and interferes with neurite formation. FEBS J. 276:6324-6337.

Hammerle B, Elizalde C, Tejedor FJ. 2008. The spatio-temporal and subcellular expression of the candidate Down syndrome gene Mnb/ Dyrk1A in the developing mouse brain suggests distinct sequential roles in neuronal development. Eur J Neurosci. 27:1061-1074.

Kaufmann WE, Moser HW. 2000. Dendritic anomalies in disorders associated with mental retardation. Cereb Cortex. 10:981-991.

Kimura R, Kamino K, Yamamoto M, Nuripa A, Kida T, Kazui H, Hashimoto R, Tanaka T, Kudo T, Yamagata H, et al. 2007. The DYRK1A gene, encoded in chromosome 21 Down syndrome critical region, bridges between beta-amyloid production and tau phosphorylation in Alzheimer disease. Hum Mol Genet. $16: 15-23$

Larkman AU. 1991. Dendritic morphology of pyramidal neurones of the visual cortex of the rat: I. Branching patterns. J Comp Neurol. 306:307-319.

Lepagnol-Bestel AM, Zvara A, Maussion G, Quignon F, Ngimbous B, Ramoz N, Imbeaud S, Loe-Mie Y, Benihoud K, Agier N, et al. 2009. DYRK1A interacts with the REST/NRSF-SWI/SNF chromatin remodelling complex to deregulate gene clusters involved in the neuronal phenotypic traits of Down syndrome. Hum Mol Genet. 18:1405-1414.

Liu CW, Lee G, Jay DG. 1999. Tau is required for neurite outgrowth and growth cone motility of chick sensory neurons. Cell Motil Cytoskeleton. 43:232-242.

Marti E, Altafaj X, Dierssen M, de la Luna S, Fotaki V, Alvarez M, PerezRiba M, Ferrer I, Estivill X. 2003. Dyrk1A expression pattern supports specific roles of this kinase in the adult central nervous system. Brain Res. 964:250-263.

Matus A. 2000. Actin-based plasticity in dendritic spines. Science. 290:754-758.

Morales R, Riss M, Wang L, Gavin R, Del Rio JA, Alcubilla R, ClaverolTinture E. 2008. Integrating multi-unit electrophysiology and plastic culture dishes for network neuroscience. Lab Chip. 8:1896-1905.

Paxinos G, Franklin K. 1997. The mouse brain in stereotaxic coordinates. San Diego (CA): Academic Press. 
Pollonini G, Gao V, Rabe A, Palminiello S, Albertini G, Alberini CM. 2008 Abnormal expression of synaptic proteins and neurotrophin-3 in the Down syndrome mouse model Ts65Dn. Neuroscience. 156:99-106.

Prinz M, Prinz B, Schulz E. 1997. The growth of non-pyramidal neurons in the primary motor cortex of man: a Golgi study. Histol Histopathol. 12:895-900.

Rahmani Z, Lopes C, Rachidi M, Delabar JM. 1998. Expression of the mnb (dyrk) protein in adult and embryonic mouse tissues. Biochem Biophys Res Commun. 253:514-518.

Ramakers GJ. 2002. Rho proteins, mental retardation and the cellular basis of cognition. Trends Neurosci. 25:191-199.

Ryoo SR, Jeong HK, Radnaabazar C, Yoo JJ, Cho HJ, Lee HW, Kim IS Cheon YH, Ahn YS, Chung SH, et al. 2007. DYRK1A-mediated hyperphosphorylation of Tau. A functional link between Down syndrome and Alzheimer disease. J Biol Chem. 282:34850-34857.

Scales TM, Lin S, Kraus M, Goold RG, Gordon-Weeks PR. 2009 Nonprimed and DYRK1A-primed GSK3 beta-phosphorylation sites on MAP1B regulate microtubule dynamics in growing axons. $J$ Cell Sci. 122:2424-2435.

Sholl DA. 1953. Dendritic organization in the neurons of the visual and motor cortices of the cat. J Anat. 87:387-406.
Star EN, Kwiatkowski DJ, Murthy VN. 2002. Rapid turnover of actin in dendritic spines and its regulation by activity. Nat Neurosci. 5:239-246.

Takashima S, Becker LE, Armstrong DL, Chan F. 1981. Abnormal neuronal development in the visual cortex of the human fetus and infant with down's syndrome. A quantitative and qualitative Golgi study. Brain Res. 225:1-21.

Takashima S, Iida K, Mito T, Arima M. 1994. Dendritic and histochemical development and ageing in patients with Down's syndrome. J Intellect Disabil Res. 38(Pt 3):265-273.

Toiber D, Azkona G, Ben-Ari S, Torán N, Soreq H, Dierssen M. 2010. Engineering DYRK1A overdosage yields Down syndromecharacteristic cortical splicing aberrations. Neurobiol Dis. 40 348-359.

Vuksic M, Petanjek Z, Rasin MR, Kostovic I. 2002. Perinatal growth of prefrontal layer $\amalg$ pyramids in Down syndrome. Pediatr Neurol. 27:36-38.

Yang EJ, Ahn YS, Chung KC. 2001. Protein kinase Dyrk1 activates cAMP response element-binding protein during neuronal differentiation in hippocampal progenitor cells. J Biol Chem. 276: 39819-39824 